\title{
ESTUDO DE ADULTERAÇÃO EM MÉIS BRASILEIROS ATRAVÉS DE RAZÃO ISOTÓPICA DO CARBONO
}

\author{
A study of adulteration in brazilian honeys by carbon isotope ratio
}

\author{
Cibele Regina de Souza-Kruliski ${ }^{1}$, Carlos Ducatti², Waldemar Gastoni Venturini Filho ${ }^{3}$, \\ Ricardo de Oliveira Orsi ${ }^{4}$, Evandro Tadeu Silva ${ }^{2}$
}

\begin{abstract}
RESUMO
Neste trabalho, objetivou-se analisar isotopicamente méis comercializados nas regiões Sul e Sudeste do Brasil, para a detecção de fraude. Foram colhidas amostras comerciais com registro no Serviço de Inspeção Federal, Estadual ou Municipal. As amostras foram submetidas à combustão no Analisador Elementar EA 1108 CHN e analisadas no espectrômetro de massas de razão isotópica DELTA-S (Finningan Mat). Os valores isotópicos $\left(\delta^{13} \mathrm{C}\right)$ dos méis in natura foram comparados aos de suas respectivas proteínas (padrão interno). Foram consideradas adulteradas as amostras cuja diferença entre o valor isotópico da proteína e do mel foi igual ou inferior a $-1 \%$. As amostras consideradas adulteradas pela análise isotópica foram submetidas a testes químicos qualitativos que não foram capazes de indicar adulteração para algumas delas. Das 61 amostras analisadas, 18,0\% encontram-se adulteradas, sendo 11,5\% na Região Sudeste e 6,5\% na Região Sul. Ao contrário dos testes químicos, a análise isotópica mostrou-se eficaz em identificar e quantificar a adulteração de méis comerciais.
\end{abstract}

Termos para indexação: Mel, adulteração, isótopos estáveis, carbono-13, IRMS.

\begin{abstract}
The aim of this study was the isotopic evaluation of honey traded in the Southern and Southeastern Brazilian regions, to detect fraud. Commercial samples, registered in the municipal, State or Federal Inspection Service, were collected and submitted to combustion in the EA 1108 CHN Elemental Analyzer and analyzed in the DELTA-S (Finningan Mat.) isotope ratio mass spectrometer. The isotopic values $\left(\delta^{13} \mathrm{C}\right)$ of in natura honey were compared to their respective proteins (internal standard). Samples whose difference between the isotopic value of protein and honey was equal or inferior to $-1 \%$ were considered adulterated. The samples considered adulterated were submitted to qualitative chemical tests which were unable to show adulteration for some of them. Among the 61 samples analyzed, $18.0 \%$ were adulterated; $11.5 \%$ in the Southeastern and $6.5 \%$ in the Southern region. Unlike chemical tests, isotopic analysis has shown to be efficient to identify and quantify adulteration in commercial honey.
\end{abstract}

Index terms: Honey, fraud, stable isotopes, carbon-13, IRMS.

(Recebido em 29 de maio de 2008 e aprovado em 17 de julho de 2009)

\section{INTRODUÇÃO}

Entre os alimentos presentes na dieta humana, o mel sempre foi alvo de adulterações (Doner, 1991). Geralmente, o processo de adulteração é feito com a adição de açúcares comerciais, como glicose comercial, solução ou xarope de sacarose, melado e solução de sacarose invertida. Porém, a forma mais utilizada de adulteração é obtida a partir do caldo de cana-de-açúcar que é levado ao fogo para concentrar o açúcar, clarificado com aditivos químicos e, em seguida, adicionado ao mel (Rossi et al., 1999).
Segundo Arauco (2005), a detecção de açúcar comercial ou açúcar invertido era feita pela quantificação de hidroximetilfurfural (HMF), composto resultante da degradação de hexoses, que pode ser encontrado no mel, em pequena quantidade. No entanto, a presença de HMF pode também ser resultado do superaquecimento do produto e estocagem prolongada. Até 1974, a detecção quantitativa e qualitativa de HMF era capaz de garantir a pureza do produto contra a principal fraude realizada até então (White Junior \& Doner, 1978a).

\footnotetext{
1Universidade Estadual Paulista/UNESP - Centro de Isótopos Estáveis Ambientais/CIE - Instituto de Biociências de Botucatu/IBB - Campus de Botucatu - Cx. P 510, 18618-000 - Botucatu, SP - cibele@ibb.unesp.br

2Universidade Estadual Paulista/UNESP - Centro de Isótopos Estáveis Ambientais/CIE - Instituto de Biociências de Botucatu/IBB - Campus de Botucatu - Botucatu, SP

${ }^{3}$ Universidade Estadual Paulista/UNESP - Faculdade de Ciências Agronômicas/FCA - Campus de Botucatu - Botucatu, SP

${ }^{4}$ Universidade Estadual Paulista/UNESP - Faculdade de Medicina Veterinária e Zootecnia/FMVZ - Campus de Botucatu - Botucatu, SP
} 
A partir daquele ano, novo tipo de adulteração começou a ser realizado nos Estados Unidos por meio da adição de HFCS (High Fructose Corn Syrup) ao mel. Trata-se de produto com baixo custo de produção, obtido a partir de tratamento enzimático do xarope de milho e que possui glicose e frutose como os principais elementos de sua composição. A adição de HFCS ao mel não pode ser detectada pela análise do hidroximetilfurfural (White Junior \& Doner, 1978a).

Na medida em que novos tipos de adulterações ocorreram, novos métodos analíticos foram desenvolvidos com intuito de detectar tais fraudes. Entre esses métodos, a análise isotópica do carbono é capaz de identificar e quantificar se o mel foi ou não adulterado com açúcar comercial ou xarope de milho (açúcares associados ao metabolismo fotossintético $\mathrm{C}_{4}$ ).

A análise isotópica de mel foi inicialmente utilizada por White Junio \& Doner (1978b), que coletaram cerca de 500 amostras de méis puros e destas, analisaram 119 amostras. Os resultados revelaram aparente homogeneidade dos dados relativos à composição isotópica, o valor médio de $\delta^{13} \mathrm{C}$ de todas as amostras foi de $-25 \%$ e o coeficiente de variação de $3,7 \%$. A partir deste trabalho, um novo método foi aprovado para o controle da qualidade do mel nos Estados Unidos (White Junior \& Doner, 1978b).

$\mathrm{Na}$ análise isotópica de mel, o espectrômetro de massa fornece os resultados em $\delta^{13} \mathrm{C}$, cuja definição matemática é dada abaixo:

$$
\delta\left(\left(_{\text {amostra, padrão }}\right)=\left[\left(\mathrm{R}_{\text {amostra }} / \mathrm{R}_{\text {padrão }}\right)-1\right]_{*} 10^{3}\right.
$$

$\delta\left({ }_{\text {amostra, padrãa }}\right)=$ enriquecimento isotópico da amostra relativo ao padrão internacional (PDB), expresso em per mil (adimensional);

$\mathrm{R}=$ razão isotópica de ${ }^{13} \mathrm{C} /{ }^{12} \mathrm{C}$, da amostra e do padrão (adimensional), respectivamente.

White Junior \& Winters (1989) propõe a utilização de um padrão interno nas análises isotópicas de mel. Sugeriram a proteína, pois esta pode ser facilmente isolada e seu valor isotópico não se altera com a adulteração do produto.

Segundo Arauco (2005), no Brasil esta técnica tem sido pouco empregada, por desconhecimento de seu potencial como método analítico por parte dos produtores, envasadores e órgãos fiscalizadores.

White Junior \& Doner (1978b) referiram-se a duas amostras de méis brasileiros, cujos resultados foram de $25,0 \%$ e $-25,6 \%$. Rossi et al. (1999) realizaram análises isotópicas em 61 amostras de méis, encontrando os seguintes valores médios: $-24,8 \%$ o para mel de laranjeira, $25,5 \%$ o para mel floral, $-25,5 \%$ o para mel de eucalipto e $-25,1 \%$ o para mel sem origem conhecida, com média geral de $-25,3 \%$. Neste trabalho, cinco amostras estavam adulteradas. Padovan et al. (2003) avaliaram 40 amostras de méis do Brasil e oito importadas (Argentina, Canadá e Estados Unidos), encontrando seis amostras de méis brasileiros adulteradas.

Arauco (2005) analisou 211 amostras de méis que foram adquiridas de apicultores de diversos estados do Brasil e constatou que $92 \%$ dos méis eram puros. A variação isotópica dos méis esteve entre $-23,79 \%$ a $-28,59 \%$ e da sua proteína variou de $-23,81 \%$ a $-28,09 \%$, atestando a idoneidade dos apiários nacionais.

Conduziu-se este trabalho, com o objetivo de analisar os méis comercializados em supermercados das Regiões Sul e Sudeste do Brasil, e indicar a porcentagem, quando houver, de adição de açúcares de plantas do ciclo fotossintético $\mathrm{C}_{4}$, por meio da análise da razão isotópica de ${ }^{13} \mathrm{C} /{ }^{12} \mathrm{C}$ e, também, comparar os resultados da análise isotópica com os de testes químicos qualitativos usados na detecção de fraude em mel.

\section{MATERIAL E MÉTODOS}

As análises isotópicas do carbono foram realizadas no Centro de Isótopos Estáveis Ambientais, Instituto de Biociências de Botucatu, UNESP. Para a análise isotópica do mel e de sua proteína, utilizou-se a metodologia oficial da Association of Official Analytical Chemists (AOAC, 2000). Algumas modificações metodológicas com relação ao procedimento de extração da proteína do mel (dobrar a quantidade dos reagentes tungstato de sódio e ácido sulfúrico) foram feitas para garantir maior quantidade de proteína extraída.

As amostras de mel e de sua proteína foram submetidas à combustão em analisador elementar Fisons Instruments EA 1108 CHN e analisadas em espectrômetro de massas de razão isotópica Finnigan Mat DELTA-S.

A porcentagem de adulteração foi determinada pela equação (2):

$$
\% \text { adulteração }=\left[\left(\delta^{13} \text { Cproteína }-\delta^{13} C \text { mel }\right)\right] /\left[\left(\delta^{13} \text { Cproteína }-\delta^{13} \text { Cadulterante }\right)\right] .100
$$


$\mathrm{Na}$ Equação 2, a diferença no valor isotópico entre a proteína e o mel ( $\delta^{13} \mathrm{C}$ proteína $-\delta^{13} \mathrm{C}$ mel $)$ de $-1 \%$ o corresponde à inclusão de $7 \%$ de produtos das plantas do ciclo fotossintético $\mathrm{C}_{4}$ (açúcares comerciais). Amostras com valores abaixo deste limite $\left(\leq-1 \%\right.$ ou $\left.\geq 7 \% \mathrm{C}_{4}\right)$ são consideradas adulteradas pela metodologia oficial (AOAC, $2000)$; consequentemente, valores acima do limite $(\geq-1 \%$ o ou $\leq 7 \% \circ \mathrm{C}_{4}$ ) indicam méis puros.

$\mathrm{Na}$ referida expressão, foi utilizado, como valor de adulterante, o valor do HFCS $(-9,7 \%$ ). No entanto, poderia ser utilizado o valor isotópico do açúcar invertido $(-12,04 \%$ ), ou ainda, o valor da glicose de milho $(-10,86 \%$ ). Optou-se pelo valor do HFCS por ser o utilizado na metodologia oficial AOAC (2000).

As amostras que foram consideradas adulteradas pelas análises isotópicas do carbono foram submetidas a testes químicos qualitativos, normalmente empregados na detecção de fraude em mel.

\section{Coleta das amostras}

Os méis foram obtidos de supermercados e estabelecimentos comerciais das regiões Sul e Sudeste do Brasil, sendo que a maioria das amostras analisadas possuía registro no Sistema de Inspeção Federal, Estadual ou Municipal.

Foram coletadas 61 amostras de mel de laranjeira, eucalipto, assa-peixe, macieira, silvestre e sem origem botânica definida (Tabela 1), com a seguinte distribuição por estado: São Paulo, 19; Rio de Janeiro, 6; Minas Gerais, 9; Espírito Santo, 2; Paraná, 7; Santa Catarina, 11; Rio Grande do Sul, 7. A origem botânica das amostras foi obtida por meio de informações contidas no rótulo.

\section{Extração da proteína do mel}

A proteína foi extraída a partir da diluição de mel em água bidestilada, seguida de clarificação em peneira (100 mesh) e homogeneização. A solução foi tratada com tungstato de sódio (10\%) e ácido sulfúrico (2\%), seguida de nova homogeneização e tratamento térmico em banhomaria $\left(80^{\circ} \mathrm{C} / 4 \mathrm{~h}\right)$. A proteína precipitada foi separada por centrifugação (3500 rpm / $5 \mathrm{~min}$ ). Essa operação foi repetida 5 vezes, utilizando água bidestilada, para a lavagem do precipitado proteico (AOAC, 2000).

\section{Procedimento utilizado para a análise isotópica do mel e sua proteína}

Foram pesados, aproximadamente, 100 a $150 \mu \mathrm{g}$ de mel e 450 a $500 \mu \mathrm{g}$ de sua respectiva proteína (seca), sendo que cada amostra foi colocada em cápsula de estanho. Essas cápsulas foram dobradas, colocadas no analisador elementar, onde ocorreu a combustão. $\mathrm{O} \mathrm{CO}_{2}$ gerado foi analisado no espectrômetro de massas de razão isotópica.

\section{Testes químicos qualitativos}

Reação de Lugol: tem como base a reação da solução de Lugol com os polímeros provenientes da hidrólise do amido contido no xarope de amido de milho hidrolisado (Marchini et al., 2004). Se o resultado for uma solução vermelha ou violeta, houve adulteração com produto derivado de amido. Reação de Lund: baseia-se na precipitação de substâncias albuminóides (proteínas e seus precursores), que são componentes naturais do mel, pela ação do ácido tânico. Se o resultado for negativo, na presença do mel puro aparecerá um precipitado cujo volume dependerá da origem floral do mel (entre 0,6 e 3,0 mL). Se o resultado for positivo, na presença do mel adulterado não haverá formação de depósito, ou ele será desprezível (Marchini et al., 2004).

Reação de Fiehe: a análise baseia-se na identificação do HMF por meio de reações químicas com resorcina em meio ácido (Marchini et al., 2004). A adulteração do mel com glicose comercial ou açúcares resulta em solução de cor vermelho-cereja.

\section{RESULTADOS E DISCUSSÃO}

Das 61 amostras analisadas, 11 foram consideradas adulteradas, segundo a metodologia oficial (AOAC, 2000). A Região Sudeste apresentou maior percentagem de fraude em relação à Região Sul. Na Região Sudeste, 7 das 36 amostras apresentaram adulteração $(19,4 \%)$, enquanto na região Sul, foram 4 das 25 amostras (16,0\%). Com relação à origem botânica dos méis, a quantidade de fraude foi: laranjeira 1 , eucalipto 2 , assa-peixe 0 , macieira 1 , silvestre 3 e sem origem botânica 4. Portanto, a maior frequência de adulteração ocorreu em méis, cujos rótulos não atestavam a sua origem botânica, sendo três deles na Região Sudeste e um na Sul.

Os resultados da variabilidade isotópica dos méis dos Estados de São Paulo (SP), Rio de Janeiro (RJ), Espírito Santo (ES), Minas Gerais (MG), Paraná (PR), Santa Catarina (SC) e Rio Grande do Sul (RS) encontram-se na Tabela 1.

Das amostras coletadas no Estado de São Paulo, seis encontraram-se adulteradas (55\% do total fraudado nas duas regiões), indicando que este é o Estado com maior número de fraudadores, merecendo maior atenção por parte dos órgãos fiscalizadores.

As amostras MG07 e SC05 não tinham mel em sua composição. Todo o carbono mensurado no espectrômetro de massa era de fonte $\mathrm{C}_{4}$ (cana-de-açúcar e/ou milho). Estas foram as fraudes mais grosseiras encontradas no presente estudo. 
Tabela 1 - Valores isotópicos $\left(\delta^{13} \mathrm{C}\right)$ das amostras de mel e de sua proteína (padrão interno) dos estados da Região Sul e Sudeste.

\begin{tabular}{|c|c|c|c|c|c|}
\hline Amostra & Origem botânica & Mel (\%o) & Proteína $(\% o)$ & Diferença & \% adulteração \\
\hline SP01 & SOB & $-24,05$ & $-24,62$ & $-0,6$ & 3,8 \\
\hline SP02 & SOB & $-25,38$ & $-24,96$ & 0,4 & 0,0 \\
\hline SP03 & $\mathrm{E}$ & $-26,42$ & $-24,86$ & 1,6 & 0,0 \\
\hline SP04 & $\mathrm{L}$ & $-24,49$ & $-24,34$ & 0,1 & 0,0 \\
\hline SP05 & S & $-25,00$ & $-25,53$ & $-0,5$ & 3,3 \\
\hline SP06 & $\mathrm{E}$ & $-27,94$ & $-26,34$ & 1,6 & 0,0 \\
\hline SP07 & $\mathrm{L}$ & $-24,74$ & $-24,80$ & $-0,1$ & 0,4 \\
\hline$\underline{\mathrm{SP} 08}$ & SOB & $\underline{-22,31}$ & $-24,24$ & $\underline{-1,9}$ & $\underline{13,3}$ \\
\hline SP09 & $\mathrm{L}$ & $-24,41$ & $-24,96$ & $-0,5$ & 3,6 \\
\hline$\underline{\text { SP10 }}$ & S & $-20,93$ & $-26,36$ & $-5,4$ & $\underline{32,6}$ \\
\hline$\overline{\mathrm{SP} 11}$ & SOB & $-25,43$ & $-25,73$ & $\overline{-0,3}$ & 1,9 \\
\hline$\underline{\mathrm{SP} 12}$ & $\mathrm{~L}$ & $-25,02$ & $-26,19$ & $-1,2$ & $\underline{7,1}$ \\
\hline$\underline{\text { SP13 }}$ & SOB & $-21,87$ & $-24,67$ & $\overline{-2,8}$ & 18,7 \\
\hline SP14 & E & $-26,57$ & $-25,26$ & 1,3 & 0,0 \\
\hline SP15 & S & $-25,57$ & $-24,98$ & 0,6 & 0,0 \\
\hline$\underline{\text { SP16 }}$ & $\mathrm{E}$ & $\underline{-26,43}$ & $\underline{-27,81}$ & $\underline{-1,4}$ & $\underline{7,6}$ \\
\hline SP17 & SOB & $-22,28$ & $-24,99$ & $-2,7$ & 17,7 \\
\hline SP18 & $\mathrm{L}$ & $-24,26$ & $-24,83$ & $-0,6$ & 3,8 \\
\hline SP19 & E & $-26,53$ & $-25,21$ & 1,3 & 0,0 \\
\hline RJ01 & SOB & $-26,19$ & $-24,94$ & 1,3 & 0,0 \\
\hline RJ02 & A & $-27,92$ & $-26,65$ & 1,3 & 0,0 \\
\hline RJ03 & S & $-27,43$ & $-26,11$ & 1,3 & 0,0 \\
\hline RJ04 & SOB & $-27,44$ & $-27,23$ & 0,2 & 0,0 \\
\hline RJ05 & $\mathrm{E}$ & $-26,49$ & $-25,63$ & 0,9 & 0,0 \\
\hline RJ06 & S & $-26,79$ & $-25,94$ & 0,9 & 0,0 \\
\hline ES01 & A & $-26,84$ & $-26,59$ & 0,3 & 0,0 \\
\hline ES02 & SOB & $-25,79$ & $-25,49$ & 0,3 & 0,0 \\
\hline MG01 & E & $-26,22$ & $-24,81$ & 1,4 & 0,0 \\
\hline MG02 & SOB & $-25,49$ & $-24,83$ & 0,7 & 0,0 \\
\hline MG03 & SOB & $-26,22$ & $-25,39$ & 0,8 & 0,0 \\
\hline MG04 & A & $-25,49$ & $-25,36$ & 0,1 & 0,0 \\
\hline MG05 & $S$ & $-26,73$ & $-25,58$ & 1,2 & 0,0 \\
\hline MG06 & A & $-26,37$ & $-25,81$ & 0,6 & 0,0 \\
\hline$\underline{\mathrm{MG} 07}$ & $\mathrm{E}$ & $\underline{-11,56}$ & $=$ & $=$ & $\underline{100}$ \\
\hline MG08 & S & $-26,72$ & $-25,23$ & 1,5 & 0,0 \\
\hline MG09 & $S$ & $-25,78$ & $-25,51$ & 0,3 & 0,0 \\
\hline PR01 & $S$ & $-27,37$ & $-26,96$ & 0,4 & 0,0 \\
\hline PR02 & SOB & $-28,35$ & $-25,55$ & 2,8 & 0,0 \\
\hline PR03 & SOB & $-27,91$ & $-27,95$ & 0,1 & 0,0 \\
\hline PR04 & SOB & $-25,71$ & $-25,65$ & 0 & 0,0 \\
\hline
\end{tabular}


Tabela 1 - Continuação...

\begin{tabular}{lccccc}
\hline PR05 & SOB & $-26,46$ & $-25,80$ & 0,7 & 0,0 \\
PR06 & SOB & $-26,42$ & $-25,84$ & 0,6 & 0,0 \\
PR07 & SOB & $-26,32$ & $-26,14$ & 0,2 & 0,0 \\
SC01 & SOB & $-25,67$ & $-25,29$ & 0,4 & 0,0 \\
SC02 & S & $-27,26$ & $-26,59$ & 0,7 & 0,0 \\
SC03 & SOB & $-25,13$ & $-25,95$ & $-0,8$ & 5,0 \\
SC04 & M & $-26,60$ & $-26,01$ & 0,6 & 0,0 \\
SC05 & M & $-11,95$ & - & - & 0,0 \\
SC06 & S & $-26,95$ & $-26,95$ & 0 & 0,0 \\
SC07 & E & $-27,17$ & $-26,45$ & 0,7 & 3,1 \\
SC08 & SOB & $-25,59$ & $-26,09$ & $-0,5$ & $\underline{57,6}$ \\
SC09 & S & $-16,25$ & $-25,14$ & $-8,9$ & 0,0 \\
SC10 & E & $-26,02$ & $-26,01$ & 0,1 & 0,0 \\
SC11 & SOB & $-26,15$ & $-25,61$ & 0,5 & 0,0 \\
RS01 & S & $-26,02$ & $-26,01$ & 0 & 5,6 \\
RS02 & E & $-25,06$ & $-25,97$ & $-0,9$ & $\underline{8,1}$ \\
RS03 & SOB & $-24,77$ & $-26,10$ & $-1,3$ & 1,5 \\
RS04 & SOB & $-25,56$ & $-25,80$ & $-0,2$ & 0,0 \\
RS05 & SOB & $-26,37$ & $-25,44$ & 0,9 & 0,0 \\
RS06 & SOB & $-26,86$ & $-26,64$ & $\underline{-8,0}$ & $\underline{57,6}$ \\
RS07 & S & $\underline{-15,59}$ & $\underline{-23,58}$ & & \\
Média \pm DP* & & $-25,02 \pm 2,05$ & $-25,68 \pm 0,64$ & & \\
\hline
\end{tabular}

*DP = Desvio Padrão; Sublinhado = amostra adulterada; $\mathrm{A}=$ assa-peixe $; \mathrm{E}=$ eucalipto;

$\mathrm{L}=$ laranjeira; $\mathrm{M}=$ macieira; $\mathrm{S}=$ silvestre; $\mathrm{SOB}=$ sem origem botânica.

Num patamar intermediário de percentual de adulteração, estão as amostras SP08 (13,3\%), SP10 $(32,6 \%)$, SP12 (7,1\%), SP13 (18,7\%), SP16 (7,6\%), SP17 (17,7), SC09 $(57,6 \%)$, RS03 $(8,1)$ e RS07 $(57,6 \%)$. Nesse caso, produtos açucarados de origem $\mathrm{C}_{4}$ foram adicionados aos méis.

As amostras que apresentaram teor de carbono $\mathrm{C}_{4}$ abaixo de $7 \%$ foram consideradas puras pela metodologia oficial (AOAC, 2000). É provável, entretanto, que essas amostras tenham sido contaminadas com carbono de fonte $\mathrm{C}_{4}$ em virtude do manejo indevido, ou seja, pela sua proximidade com a cultura de cana-de-açúcar ou alimentação das abelhas com produtos açucarados $\mathrm{C}_{4} \mathrm{em}$ período de baixa floração.

Todas as amostras dos Estados do Rio de Janeiro, Espírito Santo e Paraná não apresentaram qualquer traço de fraude.

Os méis considerados adulterados pela análise isotópica foram submetidos a três testes químicos qualitativos que podem indicar a presença de fraudes: Reação de Lugol, Reação de Lund e Reação de Fiehe (exceto as amostras SP08 e SP13, por não apresentarem quantidade suficiente). Os resultados obtidos por meio dos testes químicos qualitativos estão expressos na Tabela 2, sendo comparados com os da análise isotópica.

Os testes químicos são qualitativos, não quantificando o carbono de origem $\mathrm{C}_{4}$ que foi usado na adulteração do mel. Os resultados mostrados, na Tabela 2, indicam que esses testes apenas são eficazes para fraudes grosseiras, como foi o caso das amostras MG07, SC05 (100\% de adulteração) e RS07 (57,6\%). Para essas amostras, os três testes deram resultados positivos. Para o restante das amostras adulteradas, pelo menos um dos métodos químicos falhou na identificação da fraude. Para as amostras $\operatorname{SP} 17(17,7 \%)$ e RS03 $(8,1 \%)$, os três testes químicos falharam.

Embora os testes químicos qualitativos sejam de baixo custo e possam ser realizados por laboratórios de análises químicas básicas, eles não seriam indicados para o controle de pequenas adulterações no mel, por apresentarem menor sensibilidade. Por outro lado, as 
Tabela 2 - Resultados comparativos das análises de méis adulterados por testes químicos qualitativos e análises isotópicas. Os resultados foram apresentados como adulterados (A) e não adulterados (NA).

\begin{tabular}{ccccc}
\hline Amostras & Lugol & Fiehe & Lund & Análise Isotópica \\
\hline SP 10 & NA & A & NA & A $(32,6 \%)$ \\
SP 12 & NA & A & NA & A $(7,1 \%)$ \\
SP 16 & NA & NA & A & A $(7,6 \%)$ \\
SP 17 & NA & NA & NA & A $(17,7 \%)$ \\
MG 07 & A & A & A & A $(100 \%)$ \\
SC 05 & A & A & A & A $(100 \%)$ \\
SC 09 & A & A & NA & A $(57,6 \%)$ \\
RS 03 & NA & NA & NA & A $(8,1 \%)$ \\
RS 07 & A & A & A & A $(57,6 \%)$ \\
\hline
\end{tabular}

análises isotópicas do carbono, mesmo com custo mais elevado, conseguem detectar adulterações a partir de 3\%, quando o mel for adulterado com produtos à base de sacarose de cana-de-açúcar (Arauco, 2008). Essa análise pode ser feita em laboratórios que trabalham com espectrometria de massa de razão isotópica e deveria ser indicada como metodologia oficial para o teste de fraude em mel, por apresentar maior sensibilidade que os testes químicos qualitativos.

\section{CONCLUSÕES}

Diante dos resultados obtidos, conclui-se que a análise isotópica do carbono é uma ferramenta adequada para a identificação e quantificação de adulteração em mel. Os testes químicos qualitativos realizados neste trabalho não são capazes de identificar todos os tipos de fraude. A análise isotópica do carbono pode contribuir para melhorar a qualidade do mel comercializado no Brasil, por meio do desencorajamento da fraude por parte de produtores, distribuidores e comerciantes.

\section{REFERÊNCIAS BIBLIOGRÁFICAS}

ASSOCIATION OFFICIAL OF AGRICULTURAL CHEMISTS. Official method 998.12. C $_{4}$ : plant sugar in honey. Arlington, 2000. 1017p.

ARAUCO, E.M.R. Avaliação da qualidade do mel e atividade da enzima invertase em Apis mellifera $\mathrm{L}$. africanizadas. 2005. 109p. Tese (Doutorado em Zootecnia)-Faculdade de Medicina Veterinária e Zootecnia, Universidade Estadual Paulista, Botucatu, 2005.

DONER, L.W. Verifiying the authenticity of plant-derived material by stable isotope ratio and chromatografic methodologies. Journal of the Association of Official

Analytical Chemists, Arlington, v.74, p.14-19, 1991.

MARCHINI, L.C.; SODRÉ, G.S.; MORETI, A.C.C.C. Mel

Brasileiro: composição e normas. Ribeirão Preto: A.S.

Pinto, 2004. 111p.

PADOVAN, G.J.; JONG, D. de; RODRIGUES, L.P.; MARCHINI, J.S. Detection of adulteration of commercial honey samples by the ${ }^{13} \mathrm{C} /{ }^{12} \mathrm{C}$ isotopic ratio. Food Chemistry, Barking, v.82, p.633-636, 2003.

ROSSI, N.F.; MARTINELLI, L.A.; LACERDA, T.H.M.; CAMARGO, P.B.; VICTORIA, R.L. Análise da adulteração de méis por açúcares comerciais utilizandose a composição isotópica de carbono. Ciência e

Tecnologia de Alimentos, Campinas, v.19, n.2, p.199-200, 1999.

WHITE JUNIOR, W.; DONER, L.W. Mass spectrometric detection of high-fructose corn syrup in honey by use the of ${ }^{13} \mathrm{C} /{ }^{12} \mathrm{C}$ ratio: collaborative study. Journal of Association of Official Analytical Chemists, Arlington, v.61, p.746-750, 1978a.

WHITE JUNIOR, W.; DONER, L.W. The ${ }^{13} \mathrm{C} /{ }^{12} \mathrm{C}$ ration in honey. Journal of Apicultural Research, Cardiff, v.17, p.94-99, 1978b.

WHITE JUNIOR, W.; WINTERS, K. Honey protein as internal standard for stable carbon isotope ratio detection of adulteration of honey. Journal of the Association of Official Analytical Chemists, Arlington, v.72, p.907-911, 1989. 